\title{
Prospecção científica e tecnológica de quercetina: uso de espécies de Malpighia L. (acerola) como potencial para o tratamento de COVID-19
}

\author{
Scientific and technological prospecting of quercetin: use of Malpighia L. (acerola) species as a \\ potential for the treatment of COVID-19 \\ Prospección científica y tecnológica de quercetina: uso de la especie Malpighia L. (acerola) como \\ potencial para el tratamiento del COVID-19
}

\section{Resumo}

Quercetina é um flavonoide presente em muitos vegetais, como espécies de Malpighia L. (acerola), e tem mostrado muitas atividades farmacológicas, dentre elas o potencial antiviral e anti-inflamatório utilizado no tratamento de diversas viroses, por exemplo a COVID-19, ressaltando sua importância econômica e medicinal. Objetiva-se realizar uma prospecção científica e tecnológica acerca do flavonoide quercetina encontrado na acerola, a fim de destacar o número de publicações e de depósito de patentes em bancos de dados internacionais e evidenciar as propriedades farmacológicas e eficácia desses compostos no tratamento a COVID-19. Realizou-se uma prospecção científica e tecnológica em bases de dados de artigos e patentes internacionais sobre acerola, quercetina e COVID-19. O país com maior número de publicações foi a China $(n=25)$, e as publicações tiveram início no ano de 1967, com distribuição maior para as áreas de Medicina e Odontologia $(n=78)$. Enquanto para a prospecção tecnológica observou-se que o Japão (n=367) teve mais patentes depositadas e os depósitos começaram no ano de 2014, destacando-se A61K como a CIP mais frequente, relacionada à saúde. O Brasil não se destacou nesta pesquisa, pois a demanda tecnológica não vem sendo estimulada, o que demonstra preocupação com o país.

Palavras-chave: Acerola; Antiviral; Farmacologia.

\begin{abstract}
Quercetin is a flavonoid present in many vegetables, such as Malpighia L. (acerola), and has shown many pharmacological activities, including the antiviral and anti-inflammatory potential used in the treatment of various viruses, such as COVID-19, highlighting its economic and medicinal importance. The objective is to carry out a
\end{abstract}


scientific and technological survey of the flavonoid quercetin found in acerola, in order to highlight the number of publications and patents filed in international databases and to highlight the pharmacological properties and effectiveness of these compounds in the treatment of COVID-19. Scientific and technological research was carried out in databases of articles and international patents on acerola, quercetin and COVID-19. The country with the highest number of publications was China $(n=25)$, and publications began in 1967, with greater distribution in the areas of medicine and dentistry $(n=78)$. While for technological prospecting, it was observed that Japan $(n=367)$ had more patents filed and filings began in 2014, with A61K standing out as the most frequent CIP, related to health. Brazil did not stand out in this survey, as technological demand has not been stimulated, which demonstrates concern for the country.

Keywords: Acerola; Antiviral; Pharmacology.

\section{Resumen}

La quercetina es un flavonoide presente en muchas verduras, como la Malpighia L. (acerola), y ha mostrado muchas actividades farmacológicas, entre ellas el potencial antiviral y antiinflamatorio utilizado en el tratamiento de diversos virus, como por ejemplo el COVID-19, destacando su importancia económica y medicinal. El objetivo es realizar un estudio científico y tecnológico del flavonoide quercetina que se encuentra en la acerola, con el fin de resaltar el número de publicaciones y patentes registradas en bases de datos internacionales y resaltar las propiedades farmacológicas y la efectividad de estos compuestos en el tratamiento de COVID- 19. Se realizó investigación científica y tecnológica en bases de datos de artículos y patentes internacionales sobre acerola, quercetina y COVID19. El país con mayor número de publicaciones fue China $(n=25)$, y las publicaciones se iniciaron en 1967, con mayor distribución en las áreas de Medicina y Odontología (n=78). Mientras que para la prospección tecnológica, se observó que Japón (n=367) tuvo más patentes registradas y las solicitudes comenzaron en 2014, destacándose el A61K como el CIP más frecuente, relacionado con la salud. Brasil no se destacó en esta investigación, ya que la demanda tecnológica no llegó siendo estimulado, lo que demuestra preocupación por el país.

Palabras clave: Acerola; Antivírico; Farmacología.

\section{Introdução}

As plantas possuem importância primordial para os seres humanos, pois são capazes de produzir substâncias que podem atuar no tratamento de diversas enfermidades que atingem a humanidade. Isto ocorre devido a presença de moléculas denominadas metabólitos secundários capazes de produzir e acumular substâncias com propriedades bioativas que desempenham importante papel na forma como as plantas interagem com o meio ambiente, e tem sido bastante explorado pela farmacologia. $\mathrm{O}$ uso das plantas para fins medicinais representou os primeiros recursos terapêuticos utilizados pelo homem e faz parte da sua evolução (Brandelli, 2017).

Os metabólitos secundários são representados por três grupos: Terpenos; Compostos fenólicos e Alcalóides. O grupo dos compostos fenólicos são especialmente importantes por suas diversas funções, dentre elas a elevada capacidade antioxidante e o potencial anti-inflamatório. Este grupo tem despertado cada vez mais o interesse da indústria e dos pesquisadores (Brandelli, 2017; Brandelli \& Vieira, 2017; Saeedi-Boroujeni \& Mahmoudian-Sani, 2021) especialmente devido a pandemia ocasionada pelo vírus SARS-CoV-2.

Neste viés, os compostos fenólicos são classificados em quatro grupos: ácidos fenólicos; flavonoides (flavonóis, flavonas, isoflavonas, flavanonas, antocianidinas e quercetina); estilbenos, cujo representante mais conhecido é o resveratrol; taninos que estão divididos em dois grupos (galotaninos e elagitaninos). Entretanto, os flavonoides são os mais abundantes na dieta humana e a quercetina (3,3', $4^{\prime}, 5,6$-Pentahidroxiflavona) é considerada um dos compostos mais relevantes deste grupo (Saeedi-Boroujeni \& Mahmoudian-Sani, 2021) (Figura 1). Por isto, para investigar os efeitos biológicos dos flavonoides, a quercetina tem sido a mais estudada. 
Figura 1 - Estrutura dos flavonoides. Nas posições 3, 5, 7, 3’, 4' a quercetina tem como substituintes-OH.

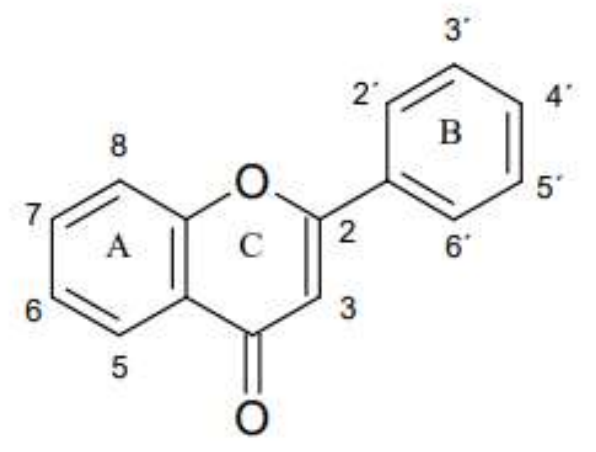

Fonte: Behling et al. (2008).

A quercetina é considerada como o principal flavonoide encontrado em alimentos com diversas atividades farmacológicas. Possui efeito anticâncer, antialérgicos, antiviral, antiprotozoários, antimicrobiano, contra artrite, doenças cardiovasculares e oculares e anti-inflamatório (Zuanazzi, Montanha \& Zucolotto, 2016; Batiha et al., 2020). O efeito antiinflamatório tem sido alvo de estudos, pois tem-se investigado a eficácia da quercetina em pacientes com sintomas graves de COVID-19, virose causada pelo SARS-CoV-2 (Athira \& James, 2021; Saeedi-Boroujeni \& Mahmoudian-Sani, 2021).

Estudos tem mostrado a presença de quercetina em algumas espécies de frutíferas, tais como Moringa oleifera Lam. (moringa) (Quijano Pérez et al., 2020), Solanum lycopersicum L. (tomate) (Leyva et al., 2021), Vitis vinifera L. (uva) (Zhang et al., 2019), Psidium guajava L. (goiaba) (Morais-Braga et al., 2017) e Malpighia spp (acerola) (Seraglio et al., 2018). No entanto, para acerola não há relatos de que este composto bioativo tenha sido investigado no tratamento da COVID-19.

O gênero Malpighia pertence à família Malpighiaceae, é constituído por cerca de 50 espécies de arbustos ou arvoretas nativas da América tropical e subtropical (Davis \& Anderson, 2010) e no Brasil são cultivadas amplamente quatro espécies devido seus frutos serem comestíveis, Malpighia glabra L., M. coccigera L., M. albiflora L. e M. emarginata DC, não endêmicas para o país. Dentre elas, M. glabra (e sinônimo M. pulnicifolia) e M. emarginata são comumente conhecidas como "acerola" (Almeida, 2020). A frutífera é importante, pois possui alto conteúdo de vitamina C, boa atividade antioxidante, dentre outras características, podendo a planta atuar como antiviral, por exemplo.

Comprovada a ineficácia dos tratamentos utilizados na COVID-19, essa poderia ser uma alternativa viável, na utilização de uma frutífera de fácil acesso para extração de substâncias com potencial bioativo, anti-inflamatório e antiviral. Por isso, é relevante apresentar dados bi prospectivos considerando-se as tecnologias produzidas e artigos como auxílio na busca por informações inovadoras acerca de determinada área com impactos sobre a sociedade, a economia e a indústria.

Dessa forma, objetiva-se por meio deste estudo realizar uma prospecção científica e tecnológica acerca do flavonoide quercetina encontrado na acerola, a fim de destacar o número de publicações e de depósito de patentes em bancos de dados internacionais e evidenciar as propriedades farmacológicas e eficácia desses compostos no tratamento a COVID-19.

\section{Metodologia}

Este trabalho é uma pesquisa documental exploratória de abordagem quantitativa (Gil, 2008). A presente pesquisa foi realizada de setembro a novembro de 2021 utilizando como palavras-chave "quercetin", "Malpighia", "acerola", "antiviral", "COVID-19". A prospecção científica consistiu na utilização das bases de dados Web of Science, Scopus e Science Direct, considerando-se a presença das palavras-chave no título e/ou resumo. 
Para melhor refinamento da pesquisa as palavras-chave foram adicionadas no campo "busca" de cada base de dados selecionada com as seguintes combinações: Quercetin AND Malpighia, quercetin AND antiviral, quercetin AND COVID-19, quercetin AND acerola, acerola AND antiviral, acerola AND COVID-19.

A análise dos artigos foi feita quanto aos países que mais publicam, ano de publicação e área de depósito. Não foi condicionada uma temporalidade para os trabalhos pesquisados, considerando-se todos aqueles apresentados pela base de dados, no intuito de investigar o período em que ocorreram as pesquisas de determinada área.

Enquanto para a prospecção tecnológica foram utilizadas a seguintes bases de patentes: United States Patent and Trademark Office (USPTO), World Intellectual Property Organization (WIPO) e European Patent Office (ESPACENET), utilizando como descritor quercetin AND COVID-19, quercetin AND acerola, acerola AND COVID-19. A análise das patentes considerou o país depositante, o ano de pedido de depósito e a Classificação Internacional de Patentes (CIP).

As patentes representam os fluxos de conhecimento que existem entre regiões, empresas e inventores, e servem para medir informações tecnológicas entre empresas, centros de pesquisas, universidades, dentre outras (Menezes, 2020).

A seleção de dados bibliográficos e patentes ocorreu devido ao número de publicações existentes, além da fidedignidade e inconstância em relação aos dados disponíveis. Ao verificar repetição de artigos nos resultados da busca entre as bases, considerava-se apenas um. Deste modo, foi adotada uma sequência analítica na obtenção dos artigos científicos em todas as buscas. A tabulação dos dados consistiu na organização individual utilizando gráficos e tabelas no Microsoft Excel 2016, bem como a caracterização dos pedidos de patente existentes até o presente momento.

\section{Resultados e Discussão}

A busca nas bases de dados teve rendimento total de 8.733 artigos indexados, destes foram selecionados apenas 2.161, conforme disposição das palavras-chave no título ou resumo. A base de maior número de artigos indexados foi Science Direct (1.151), seguido da Scopus (540) e Web of Science (470).

Ao se analisar os dados tecnológicos obteve-se o total de 755 patentes, sendo que 633 foram concedidas no banco de dados ESPACENET, 116 na USPTO (AppFT) e seis na WIPO, conforme mostra a Tabela 1.

Tabela 1- Número de artigos e patentes depositados em bancos de dados científicos e tecnológicos, respectivamente.

\begin{tabular}{lcccccc}
\hline & \multicolumn{3}{c}{ Prospecção científica } & \multicolumn{2}{c}{ Prospecção tecnológica } \\
\cline { 2 - 6 } \multicolumn{1}{c}{ DESCRITORES } & $\begin{array}{c}\text { Science } \\
\text { Direct }\end{array}$ & Scopus & $\begin{array}{c}\text { Web of } \\
\text { Science }\end{array}$ & ESPACENET & USPTO & WIPO \\
Quercetin AND Malpighia & 5 & 3 & 1 & ----- & ---- & ---- \\
Quercetin AND antiviral & 902 & 443 & 351 & ----- & ---- & ---- \\
Quercetin AND COVID-19 & 195 & 88 & 96 & 68 & 13 & 1 \\
Quercetin AND acerola & 45 & 5 & 21 & 565 & 103 & 5 \\
Acerola AND antiviral & 1 & 0 & 1 & ----- & ---- & --- \\
Acerola AND COVID-19 & 3 & 1 & 0 & 0 & 0 & 0 \\
TOTAL & $\mathbf{1 . 1 5 1}$ & $\mathbf{5 4 0}$ & $\mathbf{4 7 0}$ & $\mathbf{6 3 3}$ & $\mathbf{1 1 6}$ & $\mathbf{6}$ \\
\hline
\end{tabular}

Fonte: Autores (2021).

O maior número de registros de artigos indexados foi encontrado para os descritores "quercetin and antiviral" nos três bancos de dados utilizados, com destaque para a base Science Direct onde registrou-se 902 artigos (Tabela 1). O mesmo não 
ocorreu quando se utilizou o descritor "acerola and antiviral" (Tabela 1), apesar da literatura revelar o fruto com uma grande variedade de compostos bioativos benéficos para a saúde com elevados níveis de ácido ascórbico, carotenoides e polifenóis (Schreckinger et al., 2010; Belwal et al., 2018; Moura et al., 2018). Além disso, a quercetina está presente na sua composição.

Há relatos na literatura que confirmam os flavonoides como os fitoquímicos potencialmente benéficos na quimioterapia antiviral, podendo ser usados diretamente como medicamentos ou serem sintetizados para a produção de novos fármacos (Badshah et al., 2021). A quercetina, tem mostrado seu efeito promissor quando utilizada no combate a viroses, inibindo ou prevenindo estas. Exemplos são observados na inibição do vírus influenza, dengue tipo 2 (DENV-2), vírus da Hepatite C (HCV), dentre outros (Zandi et al., 2011; Bachmetov et al., 2012; Wu et al., 2016; Fanunza et al., 2020; Zou et al., 2020). O potencial antiviral e anti-inflamatório da quercetina justifica a existência do número elevado de artigos indexados nas bases científicas.

O SARS-CoV-2 é um beta coronavírus que pode desenvolver pneumonia inflamatória grave, considerada como fator de risco para a doença COVID-19. A quercetina também tem sido utilizada como tratamento potencial para o quadro de inflamação grave ocasionado pelo SARS CoV-2, devido sua ação antioxidante, analgésica, anti-inflamatória, inibindo a ação de inflamassoma NLRP3, um complexo proteico que é ativado mediante resposta ao quadro inflamatório, porém de forma exacerbada (Saeedi-Boroujeni \& Mahmoudian-Sani, 2021). Os achados científicos utilizando como descritor "quercetin AND COVID-19" foram expressivos com destaque para a base Science Direct onde foi encontrado o maior número de artigos indexados (195), seguida da Web of Science (96) e Scopus (87) (Tabela 1). No entanto, os achados para os descritores "acerola AND COVID-19" foram pouco expressivos quando se leva em consideração a quantidade de artigos indexados, encontrados apenas nas bases Science Direct (03) e Scopus (01) (Tabela 1). Estudos que investigam os efeitos protetores da acerola contra COVID-19 são considerados atualmente hipotéticos, conforme afirmam Günalan, Cebioğlu \& Çonak (2021).

A prospecção tecnológica revela que há expressividade quanto às patentes depositadas, especialmente em relação aos descritores "quercetin AND acerola", na base de patentes tecnológicas ESPACENET (565), seguida da USPTO (103) e WIPO (5).

\subsection{Prospecção Científica}

A China é o país com maior número de artigos indexados quando se utilizou os descritores "quercetin AND COVID19" na base Scopus, com 25 registros, representando 31\% do total de publicações, seguida da Índia com 18 artigos e 22\% das publicações, Estados Unidos com 13 e 16\% das publicações e, com apenas 1\% e 1 artigo indexado, o Brasil (Figura 2). 
Figura 2. Número de artigos indexados por país obtido, utilizando-se como descritor "quercetin AND COVID-19", no banco de dados Scopus.

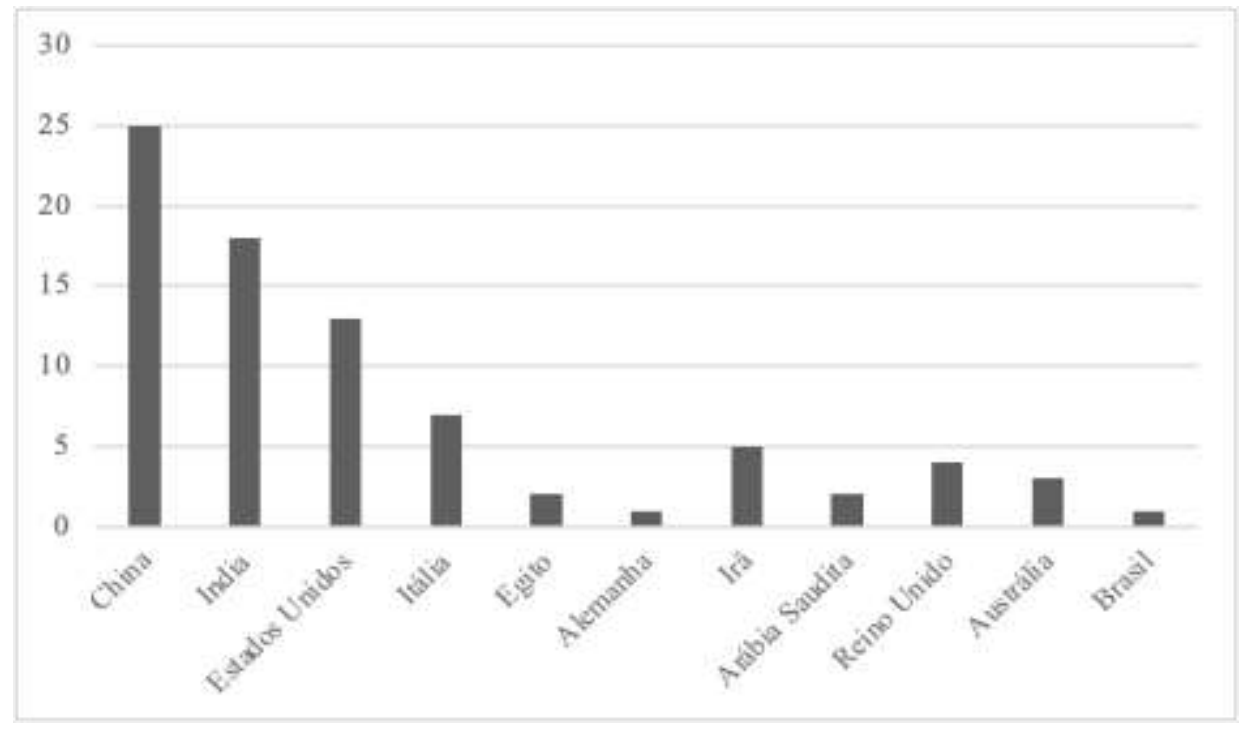

Fonte: Autores (2021).

Os dados mostram que o Brasil carece de tecnologias e mais estudos engajados em tratamentos eficazes contra a COVID-19, principalmente porque o estudo de Cavalcante et al. (2020) disponível na base de dados supracitada é de revisão bibliográfica e inclui métodos alternativos comprovados cientificamente, porém ineficazes, como o que trata do uso de hidroxicloroquina (CNN BRASIL, 2021).

Os artigos utilizando os descritores "quercetin AND acerola" na base Science Direct foram indexados a partir do ano de 1967, porém após refinamento não foi selecionado nenhum trabalho nos anos de 1967 a 2002 . Observou-se também estabilidade das publicações a partir de 2004 até 2010 (=1), com aumento pouco significativo nos demais anos (Figura 3).

O ano de maior evolução foi 2020 com sete (7) publicações, seguida de seis (6) publicações no ano de 2021 (Figura 3). As publicações de 2020 tratam principalmente sobre extração de compostos bioativos, verificação de atividade antioxidante e uso como tratamento biológico (de Oliveira et al., 2020; Girardello et al., 2020; Gomes et al., 2020; Martínez et al., 2020; Rodrigues et al., 2020; Pinto et al., 2020; Xu et al., 2020).

Figura 3. Evolução anual de artigos depositados no banco de dados Science Direct utilizando como descritores "quercetin AND acerola".

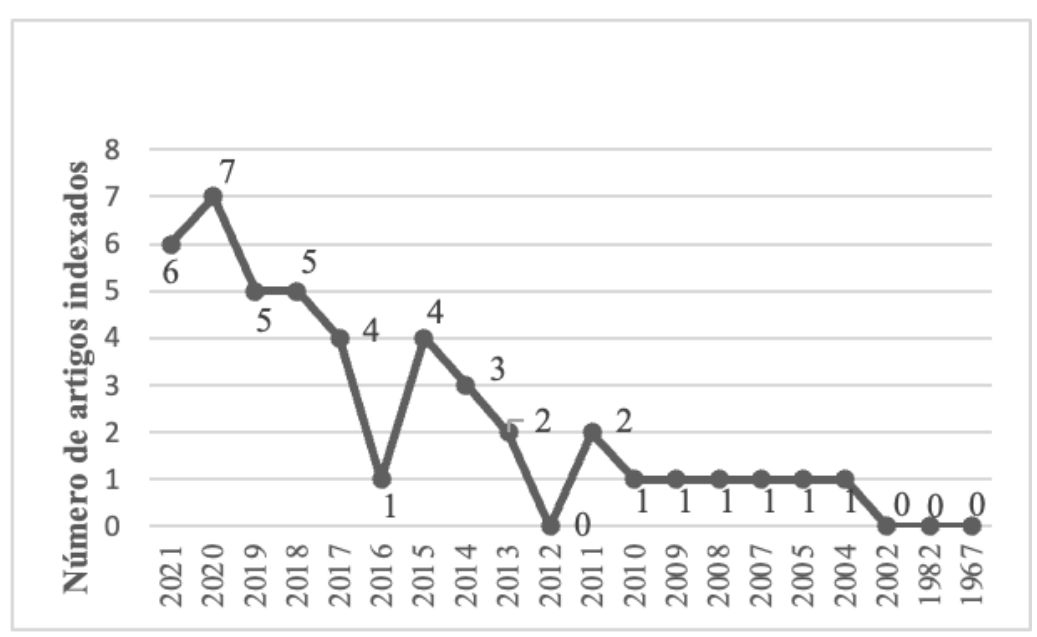

Fonte: Autores (2021). 
A este respeito, a acerola é rica em compostos bioativos, e seus efeitos benéficos são muitos, por isto representa boa opção para investimento no desenvolvimento de novos produtos, como suplementos dietéticos e alimentos funcionais. Estudo prospectivo realizado por de Carvalho Vieira et al. (2019) sugeriram que a acerola, especificamente a espécie Malpighia emarginata, teve maior destaque devido seu valor nutricional relacionado a atividade antioxidante.

Quanto ao número de manuscritos indexados por área, optou-se por utilizar os descritores "quercetin AND COVID19" na base Science Direct. As áreas mais representativas foram Medicina e Odontologia com 78 (28\%) publicações, seguida de Farmacologia, Toxicologia e Ciência Farmacêutica com 76 (28\%) e Bioquímica, Genética e Biologia Molecular com 45 (16\%) (Figura 4).

Figura 4. Distribuição das publicações indexadas por áreas na base Science Direct com "quercetin AND COVID-19".

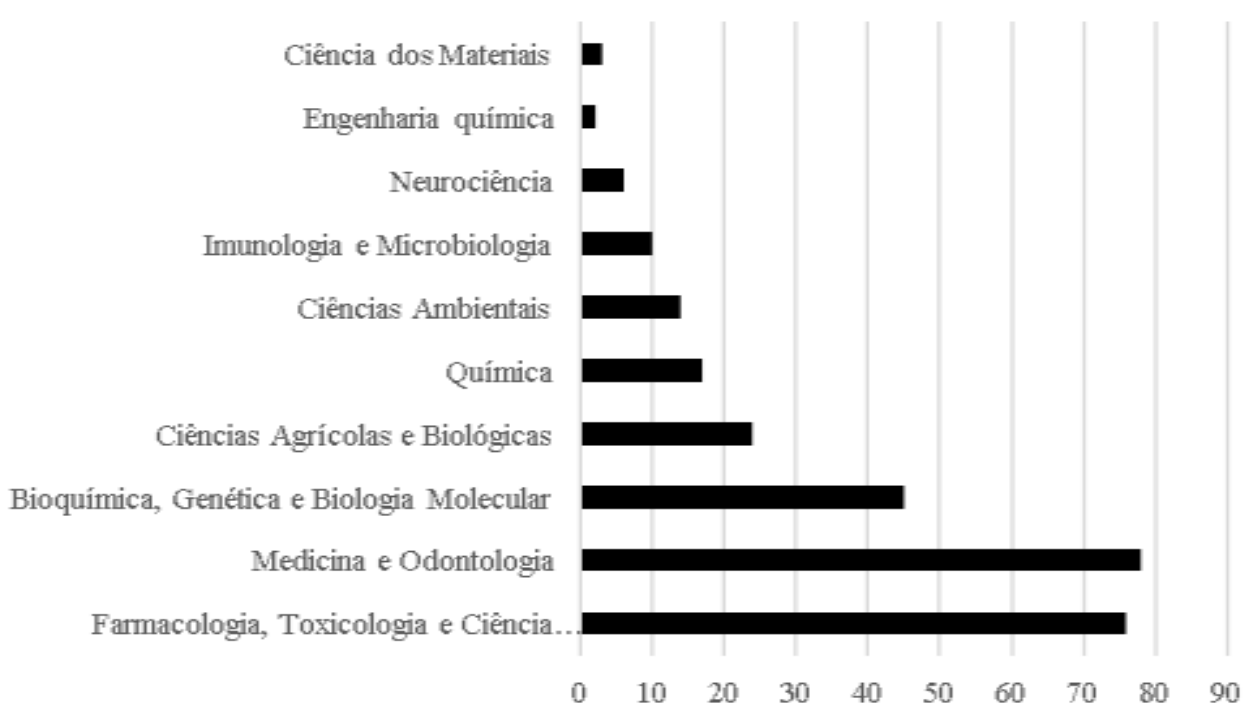

Fonte: Autores (2021).

A maior parte dos trabalhos incluídos nestas áreas, investigam o progresso do potencial medicinal e terapêutico de plantas como antiviral e anti-inflamatório, na prevenção e tratamento da COVID-19 (Shin, Oh \& Jeong, 2021; Prasansuklab et al., 2021; Lu et al., 2021; Das et al., 2021). Esses dois fatores, antiviral e anti-inflamatório, combinados, são relevantes e necessários para a produção de novos medicamentos para o tratamento eficiente da COVID-19 (Shin, Oh \& Jeong, 2021) e a quercetina estaria inclusa nestes estudos.

\subsection{Prospecção Tecnológica}

De acordo com a análise de distribuição de patentes por países nas bases selecionadas (Figura 5), constatou-se que o Japão é o maior detentor de patentes depositadas na base ESPACENET associado ao desenvolvimento de pesquisas relacionadas a quercetina e acerola. Foram 367 patentes registradas para o país que domina o ranking de um dos maiores promotores de inovação tecnológica no mundo (OMS, 2018). Os Estados Unidos da América também tiveram quantidades expressivas de depósitos de patentes registradas, aproximando-se do Japão, com 332 patentes concedidas. A Organização Mundial da Propriedade Intelectual (OMPI) com 183 depósitos, e a China com 142 registros, também tiveram boa representatividade na ESPACENET. Em relação aos depósitos na base WIPO o Japão teve apenas um (1), enquanto para a OMPI e a China não foi contabilizado nenhum depósito. 
Segundo Morais e Garcia (2012), há relevância quanto ao número de patentes depositadas, pois é um indicador importante ao se avaliar o potencial que uma região ou um país tem de transformar o conhecimento científico em um produto ou resultado tecnológico. E a inovação tecnológica é essencial para o desenvolvimento socioeconômico e sustentável de um país.

O Brasil registrou cinco patentes na ESPACENET e nenhum registro para a WIPO. Logo, observa-se que o país, apesar de apresentar mega diversidade, pois possui cerca de $20 \%$ da biodiversidade mundial, motivo pelo qual teria condições para maior inclusão no mercado de inovação de bioprodutos como os fitoterápicos (França \& Vasconcelos, 2018), encontra-se com baixa representatividade no mercado de produção farmacológica utilizando acerola e seus fitoquímicos. O banco de depósito nacional de patentes, Instituto Nacional da Propriedade Industrial (INPI), não foi selecionado para este estudo por não serem encontradas produções tecnológicas brasileiras com os descritores.

Sabe-se que o Brasil vive crise econômica com inflação alta e falta de incentivo financeiro, principalmente na realização de pesquisas científicas e tecnológicas, nesse contexto a redução de investimento na ciência e tecnologia só prolonga ainda mais o problema. Na contramão, países como Japão e Estados Unidos, mesmo quando passaram por crise econômica, como no ano de 2008, não deixaram de investir em pesquisas tecnológicas (de Moura et al., 2019).

Figura 5. Países no banco de patentes World Intellectual Property Organization (WIPO) e European Patent Office (ESPACENET) com os descritores "quercetin AND acerola".

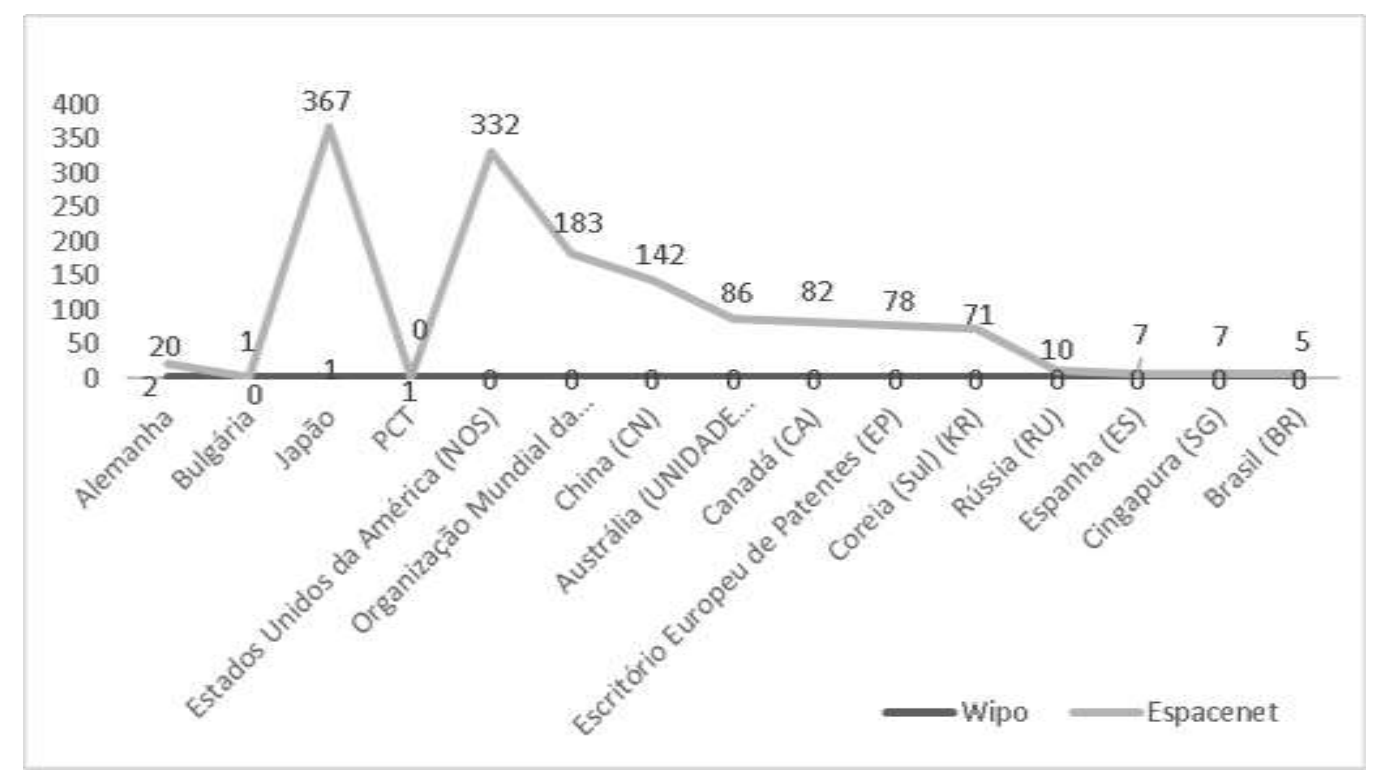

Fonte: Autores (2021).

O registro de patentes no banco de dados ESPACENET obtido com os descritores "quercetin AND COVID-19", ocorreram a partir de 2014 (Figura 6) com apenas dois depósitos de patentes intituladas "Method of treating, reducing, or alleviating a medical condition in a patient" (Método de tratamento, redução ou alívio de uma condição médica em um paciente ) que consiste em um método que inclui a administração de um paciente necessitado de uma droga biocompatível para tratar uma ou mais doenças inflamatórias do trato respiratório, reduzir os sintomas associados a elas e aliviá-las. Também foi observado a ocorrência de um depósito de patente no ano de 2016 intitulada "Method and compositions for treating coronavirus infection" (Método e composições para o tratamento da infecção por coronavírus), em que é fornecido um método de tratamento da infecção viral, como a infecção viral causada por um vírus da família Coronaviridae. Observa-se que, apesar 
dos anos de 2014 e 2016 ainda não haver iniciado o surto de COVID-19, ocasionado pelo Coronavírus, as patentes mencionadas trazem métodos relevantes para serem utilizados contra infecções virais.

Os demais registros de patentes foram observados somente a partir de 2019, ou seja, coincidindo com o início da pandemia do coronavírus, com 18 depósitos de patentes. Porém, a maior evolução de patentes ocorreu no ano de 2020 com 42 registros, fato que deve ser atribuído à procura por métodos eficazes para combater a COVID-19. O ano de 2021 teve apenas cinco depósitos na base de dados ESPACENET, além disso, este foi o único ano em que se observou ocorrência para a base de dados WIPO, com duas patentes concedidas (Figura 6).

Figura 6. Evolução anual de patentes depositadas nos bancos de bases ESPACENET e WIPO com os descritores quercetin AND COVID-19.

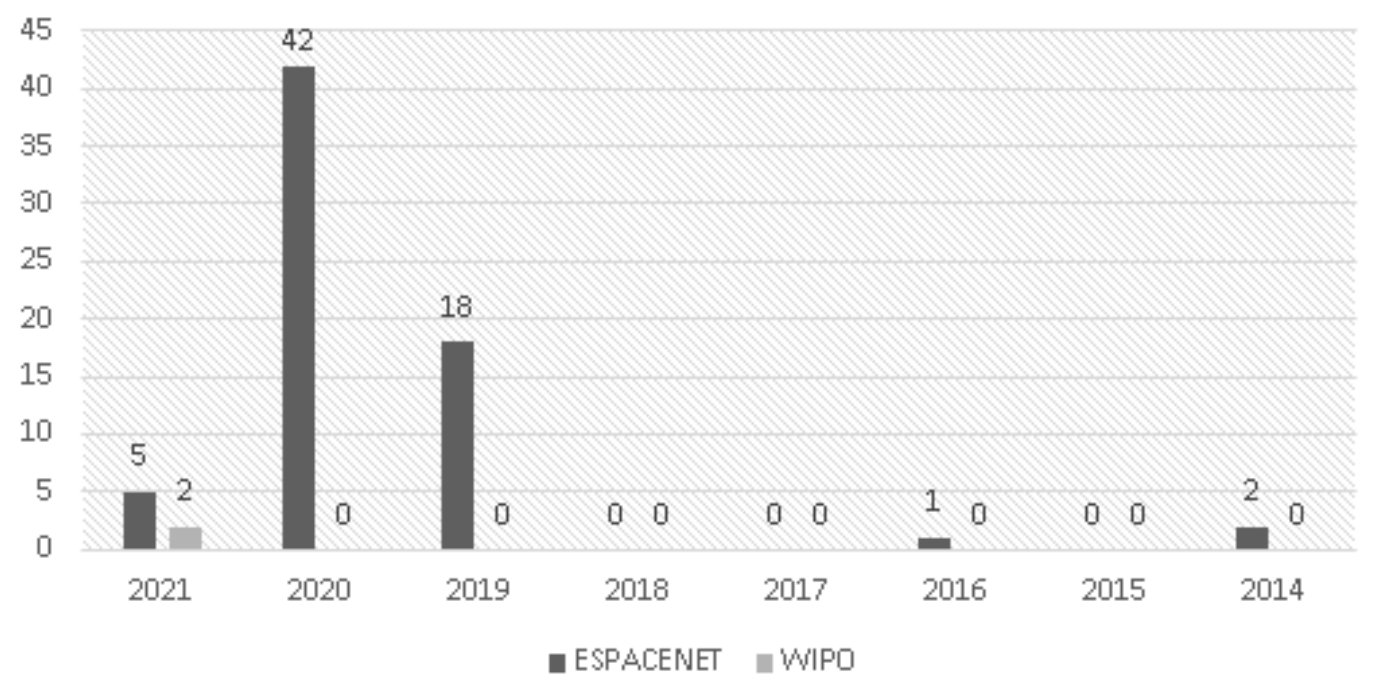

Fonte: Autores (2021).

O primeiro caso de Coronavírus ocorreu em 1960 e foi notificado como um resfriado. O vírus teria sido identificado ainda em pacientes com sintomas semelhantes à gripe em 2001 e 2002 quando não era tratado nenhum caso como fatal. Porém, em 2003 vários relatórios foram divulgados relatando que o Coronavírus havia se espalhado por diversos países como Estados Unidos, América, Hong Kong, Cingapura, Tailândia, Vietnã e em Taiwan, e reportou-se mais de 1000 pacientes mortais, identificados como casos de síndrome respiratória aguda. A COVID-19 foi identificada e isolada pela primeira vez em Wuhan, China (Kumar, Malviya \& Sharma, 2020) ainda em dezembro de 2019.

A prospecção tecnológica apresenta um formato que facilita as buscas nas bases de patentes, trazendo resultados mais precisos. A Classificação Internacional de Patentes (CIP) classifica as patentes conforme aplicação e são divididas em oito seções, 21 subseções, 120 classes, 628 subclasses e 69.000 grupos (Serafini et al., 2012).

Ao analisar as CIP provenientes das patentes das bases de dados ESPACENET e WIPO para a combinação "quercetin AND COVID-19", observou-se que cinco do total de oito CIPs selecionadas, enquadram-se na seção A (necessidades humanas) e três na seção C (química e metalurgia) (Figura 7). A subclasse A61k foi a que mais se destacou com 122 (52\%) arquivos na base ESPACENET, que corresponde a maior quantidade de patentes depositadas, e trata de preparação para fins médicos, odontológicos ou higiênicos, englobando preparações medicinais contendo ingredientes orgânicos ou inorgânicos, ativos ou não ativos, até mesmo terapia genética e preparações contendo substâncias radioativas, no âmbito do tratamento de infecções virais, especialmente a COVID-19 (ESPACENET, 2021). Na base WIPO foram contabilizados dois registros de patentes para a subclasse A61K. 
A subclasse A61P aparece em segunda colocação com 77 (33\%) registros com atividade terapêutica específica de compostos químicos ou preparações medicinais incluindo drogas para desordens dos sistemas respiratórios, nervoso, cardiovascular, urinário, endócrino, digestivo, doenças imunológicas e alérgicas, distúrbios esqueléticos, desordens dos sentidos, dentre outros, utilizados como tratamento para pacientes com COVID-19. Uma das patentes é do inventor e aplicante Hazan Sabine (2021) que propõe a administração de quatro antimicrobianos ao indivíduo, onde os antimicrobianos são compostos por quercetina, vitamina $\mathrm{C}$, vitamina $\mathrm{D}$ e zinco e visam monitorar a condição dos indivíduos durante um período de tempo pré-determinado para verificar que não se infecte com COVID-19. Foi registrada apenas uma ocorrência de patente na base WIPO para essa classificação.

Outras CIPs apareceram em menor número: A01N (n=6) e estão relacionadas à preservação de corpos de humanos ou animais ou plantas ou partes deles, biocidas, por exemplo como desinfetantes, pesticidas ou como herbicidas, repelentes ou atrativos de pragas e reguladores de crescimento de plantas. Uma das invenções fornece uma preparação medicinal e comestível chinesa que seleciona medicamentos fitoterápicos específicos e estes ingredientes podem bloquear a ligação da região receptora da proteína $\mathrm{S}$ do coronavírus à proteína receptora humana ACE2 e efetivamente inibir o vírus, além disso a invenção chinesa não tem efeitos colaterais tóxicos ao corpo humano (Dexiao et al., 2020).

A subclasse A23L também com seis patentes, corresponde a alimentos, produtos alimentícios ou bebidas não alcoólicas, sua preparação ou tratamento, por exemplo cozinha, modificação de qualidades nutritivas e tratamentos físicos. Já a subclasse A01P $(\mathrm{n}=4)$ está relacionada à atividade regulatória de compostos ou preparações químicas para biocidas, repelentes de pestes, atrativos de pestes ou de crescimento de plantas.

A subclasse C07K contém oito registros e está representada pelos peptídeos. Enquanto a subclasse $\mathrm{C} 07 \mathrm{H}(\mathrm{n}=6)$ refere-se a açúcares e seus derivados e aos nucleosídeos, nucleotídeos e ácidos nucleicos e a subclasse C11D (n= 5) que corresponde a composições de detergentes com substâncias para a produção de sabão, sabões de resina e recuperação de glicerol.

Figura 7. Classificação de acordo com a Classificação de Patentes Internacionais (CIP) utilizando os descritores "quercetin AND COVID-19" depositadas na base ESPACENET.

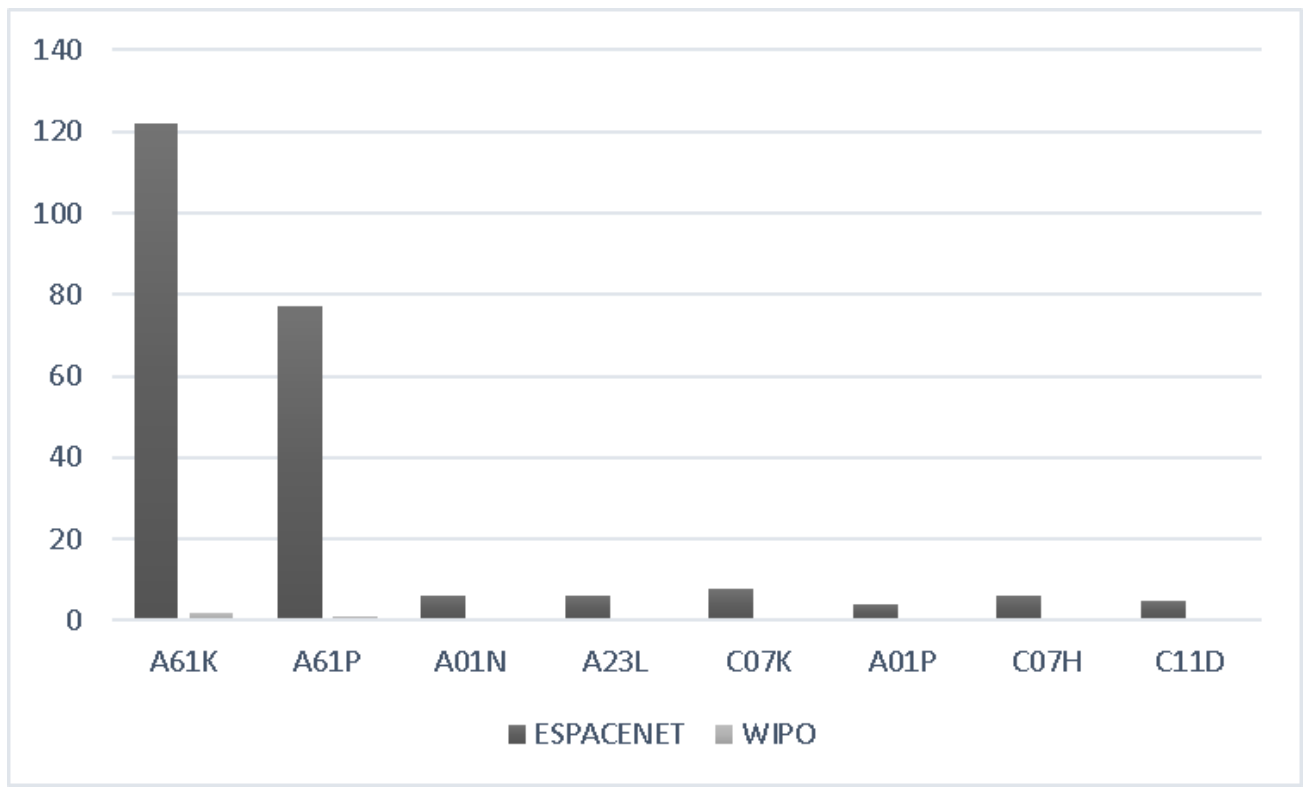

Fonte: Autores (2021). 


\section{Considerações Finais}

Observando os resultados discutidos, percebe-se que há números expressivos de publicações com a quercetina, principalmente ressaltando o potencial antiviral e anti-inflamatório que este flavonoide possui relacionado a sua atividade antioxidante. Quanto a sua relação com acerola, as publicações se restringiram ao uso da quercetina extraída de extratos dos compostos do fruto como alimento funcional, suplementos dietéticos e conservação de produtos. Além disso, apesar da acerola ter tantos benefícios para a saúde e apresentar-se como boa opção para produção tecnológica de tratamento contra a COVID19, foi possível analisar que não há relatos que a frutífera seja utilizada para tal finalidade.

O Japão é o país com maior número de patentes depositadas sobre acerola e quercetina, enquanto o Brasil teve baixa representatividade indicando que neste há a necessidade de maior produção tecnológica que possa contribuir para os avanços no aproveitamento da frutífera e de seus constituintes, já que o país é o maior produtor e exportador de acerola do mundo.

Os resultados apontam, ainda, que a quercetina tem sido bastante utilizada na indústria farmacológica como forma de aproveitar suas propriedades no tratamento a COVID-19. Nesse sentido, é relevante explorar melhor os potenciais da quercetina e investir em mais pesquisas e tecnologia que possam responder às demandas de saúde pública com a produção de medicamentos.

Este estudo prospectivo serve como embasamento para futuras produções que possam utilizar compostos fitoquímicos com propriedades medicinal e farmacológica em inovações tecnológicas. Além disto, sugere-se a ampliação de mais pesquisas como esta, porém utilizando outras bases científicas e tecnológicas como Pub Med e The Lens, respectivamente.

\section{Referências}

Almeida, R. F. (2020). Malpighia in Flora do Brasil 2020. Jardim Botânico do Rio de Janeiro. http://floradobrasil.jbrj.gov.br/reflora/floradobrasil/FB617494.

Athira Nair, D., \& James, T. J. (2020). Computational screening of phytocompounds from Moringa oleifera leaf as potential inhibitors of SARS-CoV-2 Mpro. Research Square.

Bachmetov, L., Gal-Tanamy, M., Shapira, A., Vorobeychik, M., Giterman-Galam, T., Sathiyamoorthy, P., Golan-Goldhirsh, A., Benhar, I., Tur-Kaspa, R., \& Zemel, R. (2012). Suppression of hepatitis C virus by the flavonoid quercetin is mediated by inhibition of NS3 protease activity. Journal of viral hepatitis, 19(2), e81-e88.

Badshah, S. L., Faisal, S., Muhammad, A., Poulson, B. G., Emwas, A. H., \& Jaremko, M. (2021). Antiviral activities of flavonoids. Biomedicine \& Pharmacotherapy, 140, 111596.

Batiha, G. E. S., Beshbishy, A. M., Ikram, M., Mulla, Z. S., El-Hack, M. E. A., Taha, A. E., Algammal, A. M., \& Elewa, Y. H. A. (2020). The pharmacological activity, biochemical properties, and pharmacokinetics of the major natural polyphenolic flavonoid: quercetin. Foods, $9(3), 374$.

Behling, E. V., Sendão, M. C., Francescato, H. D. C., Antunes, L. M. G., \& Bianchi, M. D. L. (2008). Flavonóide quercetina: aspectos gerais e ações biológicas. Alimentos e Nutrição Araraquara, 15(3), 285-292.

Belwal, T., Devkota, H. P., Hassan, H. A., Ahluwalia, S., Ramadan, M. F., Mocan, A., \& Atanasov, A. G. (2018). Phytopharmacology of Acerola (Malpighia spp.) and its potential as functional food. Trends in food science \& technology, 74, 99-106.

Brandelli, C. L. C. Metabolismo vegetal. In: da Cruz Monteiro, S., \& Brandelli, C. L. C. (2017). Farmacobotânica: Aspectos Teóricos e Aplicação. Artmed Editora.

Brandelli, C. L. C. \& Vieira, P. B. Substâncias bioativas. In: da Cruz Monteiro, S., \& Brandelli, C. L. C. (2017). Farmacobotânica: Aspectos Teóricos e Aplicação. Artmed Editora.

Cavalcante, M. B., Cavalcante, C. T. D. M. B., Braga, A. C. S., Andrade, D. A., Montenegro, M. A., Santos, P. A. N., Motoyama, P. V. P., Rocha, M. G., Dib, L. A., \& Júnior, E. A. (2021). COVID-19 Treatment: Drug Safety Prior to Conception and During Pregnancy and Breastfeeding. Geburtshilfe und Frauenheilkunde, 81(01), 46-60.

Das, A., Pandita, D., Jain, G. K., Agarwal, P., Grewal, A. S., Khar, R. K., \& Lather, V. (2021). Role of phytoconstituents in the management of COVID-19. Chemico-biological interactions, 109449.

Davis, C. C., \& Anderson, W. R. (2010). A complete generic phylogeny of Malpighiaceae inferred from nucleotide sequence data and morphology. American Journal of Botany, 97(12), 2031-2048.

Carvalho Vieira, T., Nascimento, M. G. P., Bittencourt, C. B., \& de Andrade, I. M. (2020). Prospecção Científica e Tecnológica de Malpighia emarginata DC. (Malpighiaceae): espécie economicamente importante do Brasil. Cadernos de Prospecção, 13(3), 862. 
França, E., \& Vasconcellos, A. G. (2019). Patentes de fitoterápicos no Brasil: uma análise do andamento dos pedidos no período de 1995-2017. Cadernos de Ciência \& Tecnologia, 35(3), 329-359.

Dexiao, Y., Yujing, L., Jinlin, Z., Yucheng, L., Huiling, L., \& Cheng, D. (2020). Patent Application N $N^{o}$ CN111773282A.

De Moura, A. M. M., Junior, R. F. G., Magnus, A. P. M., Santos, F. B., \& Scartassini, V. B. (2019). Panorama das patentes depositadas no Brasil: uma análise a partir dos maiores depositantes de patentes na base Derwent Innovations Index. Brazilian Journal of Information Science: research trends, $13(2), 59-68$.

Oliveira, S. D., Araújo, C. M., Borges, G. D. S. C., dos Santos Lima, M., Viera, V. B., Garcia, E. F., de Souza, E. L., \& de Oliveira, M. E. G. (2020). Improvement in physicochemical characteristics, bioactive compounds and antioxidant activity of acerola (Malpighia emarginata DC) and guava (Psidium guajava L.) fruit by-products fermented with potentially probiotic lactobacilli. $L W T, 134,110200$.

Fanunza, E., Iampietro, M., Distinto, S., Corona, A., Quartu, M., Maccioni, E., Horvat, B., \& Tramontano, E. (2020). Quercetin blocks Ebola virus infection by counteracting the VP24 interferon-inhibitory function. Antimicrobial agents and chemotherapy, 64(7), e00530-20.

Gil, A. C. (2008). Como elaborar projetos de pesquisa. 4. ed. São Paulo: Atlas.

Girardelo, J. R., Munari, E. L., Dallorsoleta, J. C., Cechinel, G., Goetten, A. L., Sales, L. R., Reginatto, F. H., Chaves, V. C., Smaniotto, F. A., Somacal, S., Emanuelli, T., Benech, J. C., Soldi, C., Winter, E., \& Conterato, G. M. M. (2020). Bioactive compounds, antioxidant capacity and antitumoral activity of ethanolic extracts from fruits and seeds of Eugenia involucrata DC. Food Research International, 137, 109615.

Gomes, A. C. A., da Costa Lima, M., de Oliveira, K. Á. R., dos Santos Lima, M., Magnani, M., Câmara, M. P. S., \& de Souza, E. L. (2020). Coatings with chitosan and phenolic-rich extract from acerola (Malpighia emarginata DC) or jabuticaba (Plinia jaboticaba (Vell.) Berg) processing by-product to control rot caused by Lasiodiplodia spp. in papaya (Carica papaya L.) fruit. International Journal of Food Microbiology, 331, 108694.

Günalan, E., Cebioğlu, İ. K., \& Çonak, Ö. (2021). The Popularity of the Biologically-Based Therapies During Coronavirus Pandemic Among the Google Users in the USA, UK, Germany, Italy and France. Complementary Therapies in Medicine, 58, 102682.

Hazan, S. (2021). U.S. Patent Application No. 16/953,674.

Kumar, D., Malviya, R., \& Sharma, P. K. (2020). Corona virus: a review of COVID-19. EJMO, 4(1), 8-25.

Leyva, J. F. G., Zazueta-Avitia, A., Burboa-Meza, C. Y., Ramírez-Alvarado, D., Flores-Martínez, H., \& Segura-Castruita, M. Á. (2021). Caracterización de frutos tomate (Solanum lycopersicum) en plantas colonizadas por el hongo micorrízico arbuscular Rhizopagus irregularis en condiciones de estrés salino. Acta Universitaria, 31, 1-11.

Lu, X. I. A., Yujing, S. H. I., Jie, S. U., Friedemann, T., Zhenggang, T. A. O., Lu, Y., Ling, Y., Lv, Y., Zhao, R., Geng, Z., Cui, X., Lu, H., \& Schröder, S. (2021). Shufeng Jiedu, a promising herbal therapy for moderate COVID-19: Antiviral and anti-inflammatory properties, pathways of bioactive compounds, and a clinical real-world pragmatic study. Phytomedicine, $85,153390$.

Martínez, L., Jongberg, S., Ros, G., Skibsted, L. H., \& Nieto, G. (2020). Plant derived ingredients rich in nitrates or phenolics for protection of pork against protein oxidation. Food Research International, 129, 108789.

Menezes, D. F. N. (2020). Bio e nanotecnologia: análise da convergência tecnológica pelas patentes. Revista Jurídica Luso-Brasileira, 6(5), 557-578.

Moura, C. F., Oliveira, L. D. S., de Souza, K. O., da Franca, L. G., Ribeiro, L. B., de Souza, P. A., \& de Miranda, M. R. (2018). Acerola—Malpighia emarginata. In Exotic fruits (pp. 7-14). Academic Press.

Morais, S. M. P. D., \& Garcia, J. C. R. (2013). Inovação tecnológica em publicações brasileiras da ciência da informação.

Morais-Braga, M. F., Carneiro, J. N., Machado, A. J., Sales, D. L., Dos Santos, A. T., Boligon, A. A., Athayde, M. L., Menezes, I. R. A., Souza, D. S. L., Costa, J. G. M., \& Coutinho, H. D. (2017). Phenolic composition and medicinal usage of Psidium guajava Linn.: Antifungal activity or inhibition of virulence? Saudi Journal of Biological Sciences, 24(2), 302-313.

OMS - Organização Mundial da Saúde. (2018). Global Innovation Index 2018. https://www.wipo.int/pressroom/en/ar ticles/2018/article_0005.html.

CNN BRASIL- Cable News Network. (2021). https://www.cnnbrasil.com.br/saude/entenda-as-recomendacoes-das-instituicoes-de-saude-contra-o-uso-decloroquina/

Pinto, M., Benfeito, S., Fernandes, C., \& Borges, F. (2020). Antioxidant therapy, oxidative stress, and blood-brain barrier: the road of dietary antioxidants. In Oxidative Stress and Dietary Antioxidants in Neurological Diseases (pp. 125-141). Academic Press.

Prasansuklab, A., Theerasri, A., Rangsinth, P., Sillapachaiyaporn, C., Chuchawankul, S., \& Tencomnao, T. (2020). Anti-COVID-19 drug candidates: a review on potential biological activities of natural products in the management of new Coronavirus infection. Journal of traditional and complementary medicine.

Quijano Pérez, J. E., Segura Cobos, D., García Pineda, M., Omaña Molina, M. A., \& Guzmán Hernández, E. A. (2020). Efecto hipoglucemiante y nefroprotector de Olea europea, Moringa oleifera y Chicorium intibus var en un modelo experimental de diabetes mellitus. https://revistatediq. azc. uam. $\mathrm{mx} /$ Docs/Revista_TeDIQ_2020. pdf.

Rodrigues, L. M., Romanini, E. B., Silva, E., Pilau, E. J., da Costa, S. C., \& Madrona, G. S. (2020). Camu-camu bioactive compounds extraction by ecofriendly sequential processes (ultrasound assisted extraction and reverse osmosis). Ultrasonics sonochemistry, 64, 105017.

Saeedi-Boroujeni, A., \& Mahmoudian-Sani, M. R. (2021). Anti-inflammatory potential of Quercetin in COVID-19 treatment. Journal of Inflammation, 18(1), $1-9$.

Serafini, M. R., Quintans, J. D. S. S., Antoniolli, Â. R., dos Santos, M. R. V., \& Quintans-Junior, L. J. (2012). Mapeamento de tecnologias patenteáveis com o uso da hecogenina. Revista geintec-gestão inovação e tecnologias, 2(5), 427-435. 
Research, Society and Development, v. 11, n. 1, e19711124715, 2022

(CC BY 4.0) | ISSN 2525-3409 | DOI: http://dx.doi.org/10.33448/rsd-v11i1.24715

Seraglio, S. K. T., Schulz, M., Nehring, P., Della Betta, F., Valese, A. C., Daguer, H., Gonzaga, L. V., Fett, R., \& Costa, A. C. O. (2018). Determinação de compostos fenólicos por LC-MS/MS e capacidade antioxidante de acerola em três estádios de maturação comestíveis. In Revista do Congresso Sul Brasileiro de Engenharia de Alimentos, 4 (1).

Schreckinger, M. E., Lotton, J., Lila, M. A., \& de Mejia, E. G. (2010). Berries from South America: a comprehensive review on chemistry, health potential, and commercialization. Journal of medicinal food, 13(2), 233-246.

Shin, J. A., Oh, S., \& Jeong, J. M. (2021). The potential of BEN815 as an anti-inflammatory, antiviral and antioxidant agent for the treatment of COVID-19. Phytomedicine Plus, 1(4), 100058.

Wu, W., Li, R., Li, X., He, J., Jiang, S., Liu, S., \& Yang, J. (2016). Quercetin as an antiviral agent inhibits influenza A virus (IAV) entry. Viruses, 8(1), 6.

Xu, M., Shen, C., Zheng, H., Xu, Y., Xue, C., Zhu, B., \& Hu, J. (2020). Metabolomic analysis of acerola cherry (Malpighia emarginata) fruit during ripening development via UPLC-Q-TOF and contribution to the antioxidant activity. Food Research International, 130, 108915.

Zhang, J. R., Trossat-Magnin, C., Bathany, K., Delrot, S., \& Chaudière, J. (2019). Transformação oxidativa de leucociaanidina por anthocyanidin synthase de Vitis vinifera leva apenas à quercetina. Revista de química agrícola e alimentar, 67(13), 3595-3604.

Zandi, K., Teoh, B. T., Sam, S. S., Wong, P. F., Mustafa, M. R., \& AbuBakar, S. (2011). Antiviral activity of four types of bioflavonoid against dengue virus type-2. Virology journal, 8(1), 1-11.

Zou, M., Liu, H., Li, J., Yao, X., Chen, Y., Ke, C., \& Liu, S. (2020). Structure-activity relationship of flavonoid bifunctional inhibitors against Zika virus infection. Biochemical pharmacology, 177, 113962.

Zuanazzi, J. A. S., Montanha, J. A., \& Zucolotto, S. M. Flavonoides. In: Simões, C. M. O., Schenkel, E. P., de Mello, J. C. P., Mentz, L. A., \& Petrovick, P. R. (2016). Farmacognosia: do produto natural ao medicamento. Artmed Editora. 